\title{
Method for showing human spermatogenesis using Arachis hypogaea (AHA) lectin
}

\author{
CAROLYN J P JONES, E W BENBOW, W FIONA KNOX, R W STODDART Pathology \\ Department, University of Manchester, Manchester
}

SUMMARY Using biotinylated Arachis hypogaea agglutinin (AHA) or peanut lectin (PNA) and an avidin-peroxidase procedure, the various stages of spermatid development were visualised with great clarity; a light haematoxylin counterstain permitted the easy recognition of spermatogenic cells in the same section. This method is particularly useful in the interpretation of poorly fixed material and may be helpful in studies of cyclical maturation of spermatozoa, irrespective of whether the material is obtained at biopsy or necropsy.

Difficulties may be experienced in the interpretation of testicular biopsy specimens because of factors such as inadequate specimen size, trauma during handling, and fixation in an inappropriate fixative. 'We describe a simple method for the demonstration of the acrosomal apparatus which can be used as a marker of spermatid development, thus facilitating the evaluation of spermatogenesis. This technique utilises the binding of the lectin Arachis hypogaea agglutinin (AHA), also known as peanut agglutinin (PNA).

\section{Material and methods}

Paraffin wax sections $2-4 \mu \mathrm{m}$ thick from 18 unselected human testicular biopsy specimens fixed in Bouin's solution and eight formalin fixed necropsy specimens were treated in the following manner:

1 Sections were dewaxed and taken to absolute alcohol.

2 Endogenous peroxidase activity was blocked with absolute methanol containing $0.4 \%(\mathrm{v} / \mathrm{v})$ hydrochloric acid (N/l) and $0.5 \%(v / v)$ hydrogen peroxide (100 volumes) for 30 minutes at room temperature.

3 Wash in $0.05 \mathrm{M}$ Tris buffered saline (TBS), pH 7.6.

4 Treat with $0 \cdot 1 \%(\mathrm{w} / \mathrm{v})$ crude trypsin (Sigma type II) in $0.05 \mathrm{M}$ TBS (pH 7.6) containing $0.09 \mathrm{M}$ $(\mathrm{w} / \mathrm{v})$ calcium chloride for 15 minutes at $37^{\circ} \mathrm{C}$. (Under these conditions its effect is attributable to chymotrypsin in aldehyde-fixed material ${ }^{2}$ ).

5 Wash in 0.05 M TBS (pH 7.6) for 15 minutes (three changes).

6 Incubate in $10 \mu \mathrm{g} / \mathrm{ml}$ biotinylated Arachis

Accepted for publication 7 April 1988 hypogaea agglutinin (AHA; Sigma) in 0.05 M TBS (pH 7.6) containing $1 \mathrm{mM}$ calcium chloride for $\mathbf{3 0}$ minutes at room temperature in a humidity chamber.

7 Wash for 10 minutes (two changes) in $0.05 \mathrm{M}$ TBS (pH 7.6) containing $1 \mathrm{mM}$ calcium chloride.

8 Incubate in $5 \mu \mathrm{g} / \mathrm{ml}$ avidin peroxidase (Sigma) in $0.125 \mathrm{M}$ TBS (pH 7.6) containing $0.347 \mathrm{M}$ sodium chloride for 60 minutes at room temperature in a humidity chamber; this high molarity buffer reduces background staining. ${ }^{3}$

9 Wash in $0.05 \mathrm{M}$ TBS ( $\mathrm{pH} \mathrm{7.6)}$ ) for 15 minutes (three changes).

10 Show sites of lectin binding with $0.05 \%(w / v)$ diaminobenzidine tetrahydrochloride dihydrate (Aldrich Chemical Company, Gillingham, Kent) in $0.05 \mathrm{M}$ TBS $\mathrm{pH} 7.6$ with $0.015 \%$ hydrogen peroxide ( $\mathrm{v} / \mathrm{v}$ by dilution from 100 volumes) for five minutes.

11 Wash, then counterstain lightly with Harris's haematoxylin.

12 Wash, dehydrate, clear and mount in XAM (Gurr, BDH, Poole, Dorset).

\section{Results}

In both biopsy (fig 1) and necropsy material (fig 2) peanut lectin (AHA) bound to the acrosomes of developing spermatids and, to a lesser degree, to mature spermatozoa, in a chemically highly specific and morphologically selective manner. In the earliest stage it appears as an intense dark-brown paranuclear "dot" (the acrosomal granule); later stages appear as a crescent-shaped "cap" over the anterior edge of the spermatid nucleus. As the nucleus elongates during spermatogenesis the acrosome progressively blends 


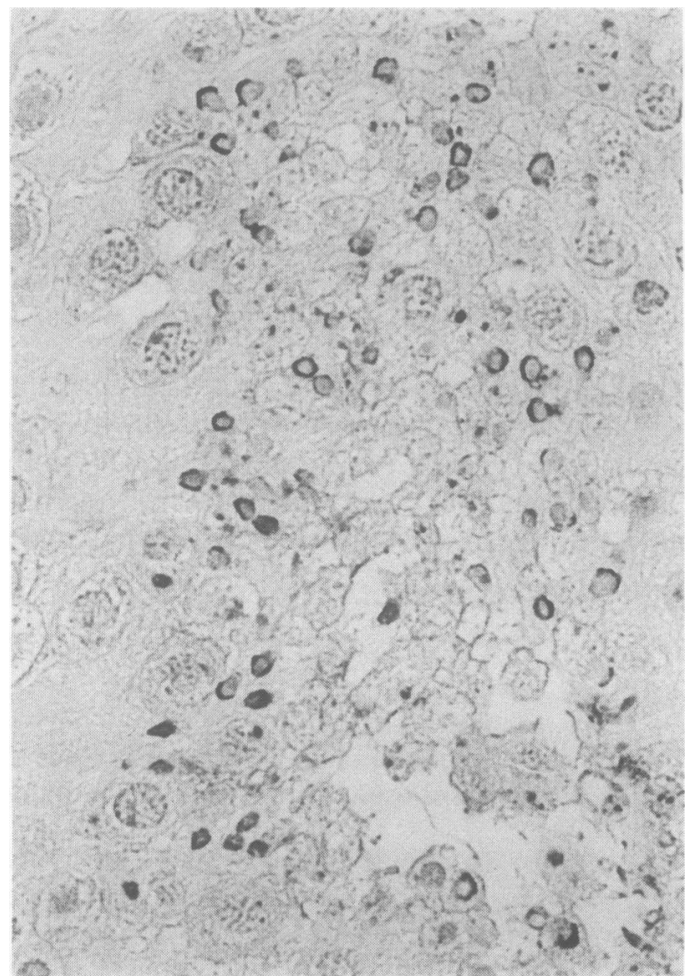

Fig 1 Human testicular biopsy specimen from a case of germ cell arrest, fixed in Bouin's solution, and stained with peanut lectin, showing mainly crescent-shaped acrosomes on developing spermatozoa.

with the apex of the head cap. Binding of the lectin gradually becomes weaker as the spermatozoa reach their final mature form. The developmental sequence may be viewed with great clarity, while the use of a haematoxylin counterstain facilitates easy recognition of the nuclear configurations of germinal epithelium, spermatozoa, and spermatocytes. With Bouin's fixative there was, in some specimens, weak cytoplasmic background staining, but this was not found in formalin fixed material. It was particularly pronounced in seminiferous tubules that showed sloughing of cells into the lumen, although it never reached a level where acrosomal staining was obscured.

\section{Discussion}

The acrosome is a structure rich in carbohydrates, which has been shown to bind many lectins in a variety of mammalian species. ${ }^{45}$ In our experience, however, only that derived from Arachis hypogaea (AHA or PNA) used under the conditions above, shows complete selectivity for the acrosome in the human testis. The method which we describe may have potential use in the assessment of spermatogenesis and, particularly, in the evaluation of spermatid development.

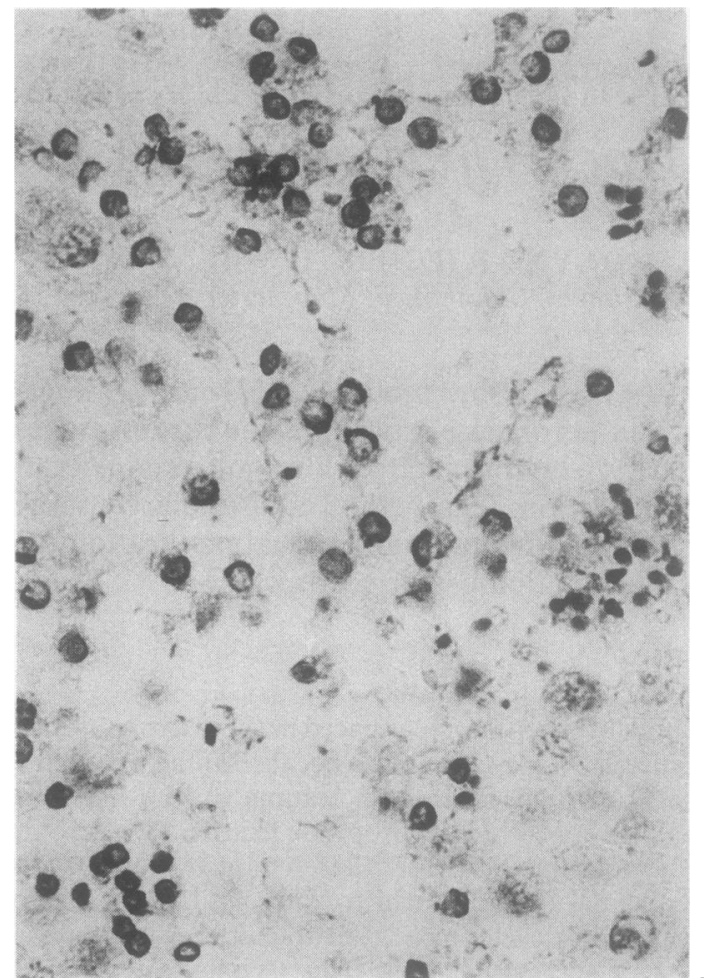

Fig 2 Formalin fixed necropsy specimen of testis stained with peanut lectin (42 hours after death) showing good preservation of acrosomal apparatus.

It could be especially helpful in studies of the cyclical maturation within the seminiferous tubule because both nuclear detail and acrosomal structures are well visualised. Although its main application is likely to be in research, it may aid the examination of clinical material, especially in conditions such as maturation arrest; in particular, it may assist when fixation has been late or inadequate, for it greatly enhanced the identifiability of the cells in the formalin fixed material, even though it was derived from necropsy material.

\section{References}

1 Ansell ID. Atlas of Male Reproductive Pathology. Lancaster: MTP Press Ltd, 1985:21.

2 Jeffrey IJM, Mosley SM, Jones CJP, Stoddart RW. Proteolysis and lectin histochemistry. Histochem $J$ 1987;19:269-75.

3 Jones CJP, Mosley SM, Jeffrey IJM, Stoddart RW. Elimination of the non-specific binding of avidin to tissue sections. Histochem $J$ 1987;19:264-8.

4 Lee M-C, Damjanov I. Lectin binding sites on human sperm and spermatogenic cells. Anat Rec 1985;212:282-7.

5 Arya M, Vanha-Perttula T. Comparison of lectin-staining pattern in testis and epididymis of gerbil, guinea pig, mouse and nutria. Am J Anat 1986;175:449-69.

Requests for reprints to: Dr Carolyn J P Jones, Department of Pathology, University of Manchester, Stopford Building, Oxford Road, Manchester M13 9PT, England. 